\title{
Relationships between serum selenium and zinc concentrations versus profibrotic and proangiogenic cytokines (FGF-19 and endoglin) in patients with alcoholic liver cirrhosis
}

\author{
Andrzej Prystupa ${ }^{1, A-B, D-F}$, Paweł Kicińskii, ${ }^{2, C-F}$, Dorota Luchowska-Kocot ${ }^{3, B}$, Anna Błażewicz ${ }^{4, B}$, \\ Ewa Kurys-Denis ${ }^{5, B}$, Jarosław Niedziałek ${ }^{6, C}$, Jarosław Sak ${ }^{7, A}$, Lech Panasiuk ${ }^{8, F}$ \\ ${ }^{1}$ Department of Internal Medicine, Medical University of Lublin, Lublin, Poland \\ 2 Department of Family Medicine, Medical University of Lublin, Lublin, Poland \\ ${ }^{3}$ Department of Medical Chemistry, Medical University of Lublin, Lublin, Poland \\ ${ }^{4}$ Department of Analytical Chemistry, Medical University of Lublin, Lublin, Poland \\ ${ }^{5}$ II Department of Radiology, Medical University of Lublin, Lublin, Poland \\ ${ }^{6}$ Individual Medical Practice, Lublin, Poland \\ ${ }^{7}$ Department of Ethics and Human Philosophy, Medical University of Lublin, Lublin, Poland \\ ${ }^{8}$ Institute of Rural Medicine, Lublin, Poland \\ A - Research concept and design, B - Collection and/or assembly of data, C - Data analysis and interpretation, \\ $D$ - Writing the article, E - Critical revision of the article, F - Final approval of article
}

\begin{abstract}
Prystupa A, Kiciński P, Luchowska-Kocot D, Błażewicz A, Kurys-Denis E, Niedziałek J, Sak J, Panasiuk L. Relationships between serum selenium and zinc concentrations versus profibrotic and proangiogenic cytokines (FGF-19 and endoglin) in patients with alcoholic liver cirrhosis. Ann Agric Env Med. 2017; 24(3): 544-548. doi: 10.26444/aaem/76937
\end{abstract}

\section{Abstract}

Introduction and objective. Liver cirrhosis is a disease involving the liver parenchyma, which is characterised by fibrosis. and impaired architectonics of the parenchyma with regenerative nodules. The aim of the study was to determine the relationship between stage of alcoholic liver cirrhosis, concentrations of selenium, zinc and profibrotic and proangiogenic cytokines (FGF-19, ENG).

Materials and method. The study included 99 patients with alcoholic cirrhosis and 20 healthy subjects. Ion chromatography with UV/VIS detection was used for determination of zinc ions in the previously mineralized serum samples. The measurements of selenium were performed with the ContrAA700 high-resolution continuum source graphite tube atomic absorption spectrometer. ELISA was used to determine concentration of FGF-19 and ENG in serum samples.

Results. Concentrations of zinc and selenium were significantly decreased in cirrhotic patients ( $p<0.001$ for both). The highest concentration of FGF-19 was found in Child-Pugh stage C liver cirrhosis patients $(806.9 \pm 650.3 \mathrm{pg} / \mathrm{ml})$, and was significantly higher than observed in controls ( $p=0.005)$ and stage A patients (compensated cirrhosis) $(p=0.02)$. The highest concentration of ENG was demonstrated in the control group $(3.24 \pm 148 \mathrm{ng} / \mathrm{ml})$ while the lowest in patients with decompensated cirrhosis $(7.32 \pm 5.39 \mathrm{ng} / \mathrm{ml}$ and $7.92 \pm 4.18 \mathrm{ng} / \mathrm{ml}$ for stage $B$ and $C ; p=0.03$ and $p=0.02$, respectively). The use of the multiple-variable model demonstrated that the independent factors affecting the concentration of ENG were the concentration of bilirubin $(p=0.02)$, INR ( $p=0.01)$ and duration of alcohol abuse $(p=0.02)$. The independent determinants of FGF-19 concentrations were found to be the stage (severity) of liver cirrhosis ( $p=0.04)$ and INR ( $p=0.03)$.

Conclusions. Concentrations of zinc and selenium in serum of patients with alcoholic liver cirrhosis are not independently related to concentrations of FGF-19 and ENG.

\section{Key words}

liver cirrhosis, alcohol, selenium, zinc, fibroblast growth factor-19, endoglin

\section{INTRODUCTION}

Liver cirrhosis is a disease involving the liver parenchyma, which is characterised by fibrosis and impaired architectonics of the parenchyma with regenerative nodules. Besides infections with hepatotropic viruses, alcohol abuse is the major cause of liver cirrhosis [1]. Europe is ranked amongst the geographical regions with the highest alcohol consumption. Alcohol consumption decreased in the 1990s to re-increase and stabilise at higher levels in 2004-2006, varying greatly

Address for correspondence: Andrzej Prystupa, Department of Internal Medicine Medical University of Lublin, Lublin, Poland

e-mail: aprystup@wp.pl

Received: 31.08.2017; accepted: 12.09.2017; first published: 16.09.2017 depending on the country [2]. In Poland, the mortality due to liver cirrhosis increased from 5.14/100,000 in 1980 to $7.60 / 100,000$ in 2010 [3].

Various cells are involved in the pathogenesis of liver cirrhosis, including hepatocytes, Browicz-Kupfer cells, liver sinusoidal endothelial cells and stellate cells. Activation of stellate cells and promotion of fibrogenesis are highly affected by the network of pro-inflammatory, profibrotic and proangiogenic cytokines, including tumour necrosis factor $\alpha$ (TNF- $\alpha$ ), platelet-derived growth factor (PDGF), transforming growth factor $\beta$ (TGF- $\beta$ ), fibroblast growth factor 19 (FGF-19), among others [4].

Endoglin (ENG, known also as the cluster of differentiation 105, CD105) acts as a TGF- $\beta$ transmembranous coreceptor 
and its low expression has been found in resting endothelial cells. Physiologically, ENG is responsible for the regulation of cell processes associated with angiogenesis. Moreover, it is actively involved in the proliferation, migration, adhesion and formation of the vascular cytoskeleton, as well as in apoptosis and transformation of the extracellular matrix [5]. The expression of ENG is markedly higher in the proliferating endothelium and the blood vessels of neoplastic tumours. Therefore, it is considered essential for neoangiogenesis, tumour growth and formation of metastases [6]. ENG has been demonstrated to stimulate hepatic stellate cells and thus to induce the profibrogenic effects in the liver $[7,8]$.

The physiological role of FGF-19 is associated with the regulation of synthesis of bile acids. FGF-19 is involved in the pathogenesis of primary liver cirrhosis [9]. The FGF-19mediated activation of mitogen-activated protein kinase and the $\beta$-catenin pathway can be associated with progression of hepatocellular carcinoma [10].

The studies available in literature demonstrate the deficiencies of microelements, such as zinc and selenium, in alcoholics and patients with liver cirrhosis [11]. Selenium shows antioxidative effects and occurs in the form of selenoprotein as a cofactor of many enzymes. Zinc, on the other hand, determines the normal activity of about 300 metalloenzymes. Recent studies indicate that zinc deficiencies are connected with the progression of liver cirrhosis [12]. According to some authors, the deficiencies of the above elements are likely to be associated with the pathogenesis of liver cirrhosis, which can justify their supplementation [13]. The exact molecular mechanisms of hepatoprotective effects of zinc and selenium are not fully elucidated.

\section{OBJECTIVES}

The aim of the study was to determine the relationship between the stage of alcoholic liver cirrhosis, concentrations of selenium, zinc and profibrotic and proangiogenic cytokines (FGF-19, ENG).

\section{MATERIALS AND METHOD}

\section{Patients}

The study included 99 patients with alcoholic cirrhosis from Lublin Region of eastern Poland). All patients enrolled in the study had consumed large amounts of alcohol over a long period of time. Liver cirrhosis was diagnosed based on clinical features, history of heavy alcohol consumption, laboratory tests and abdominal ultrasonography. The exclusion criteria were alcoholic hepatitis, viral and autoimmune diseases. The severity of liver cirrhosis was evaluated according to the Child-Turcotte-Pugh criteria (Child-Pugh score) [14]. Based on these, the patients were assigned to one of three groups: 1 ) Pugh-Child (P-Ch) A - 29 with stage A, 2) P-Ch B - 26 with stage $\mathrm{B}$ and 3) $\mathrm{P}-\mathrm{Ch} \mathrm{C}-34$ with stage $\mathrm{C}$ of liver cirrhosis. The control group consisted of 20 healthy individuals without liver disease who did not abuse alcohol. None of the patients and healthy participants received mineral supplements. In the control group, both clinical assessment and laboratory tests were used to exclude underlying liver diseases. There were no significant age- or gender-related differences in the subgroups (Table 1). Detailed demographic, clinical and
Table 1. Demographic and clinical characteristics of study and control groups

\begin{tabular}{|c|c|c|c|c|}
\hline & \multirow{2}{*}{$\begin{array}{l}\text { Control group } \\
\qquad(n=20)\end{array}$} & \multicolumn{3}{|c|}{ Alcoholic liver cirrhosis ( $n=99$ ) } \\
\hline & & $\begin{array}{l}\text { P-Ch A } \\
(n=29)\end{array}$ & $\begin{array}{l}\text { P-Ch B } \\
(n=36)\end{array}$ & $\begin{array}{l}\text { P-Ch C } \\
(n=34)\end{array}$ \\
\hline Age (years) & $48.9 \pm 15.1$ & $53.3 \pm 12.3$ & $54.6 \pm 11.0$ & $56.9 \pm 7.7$ \\
\hline Percentage of males & $65 \%$ & $72.4 \%$ & $66.7 \%$ & $58.8 \%$ \\
\hline Height (cm) & $170.4 \pm 6.6$ & $171.8 \pm 7.8$ & $175.3 \pm 8.4$ & $173.4 \pm 6.9$ \\
\hline Body mass (kg) & $67.8 \pm 7.8$ & $68.1 \pm 14.8$ & $71.5 \pm 13.2$ & $70.2 \pm 12.8$ \\
\hline Time of alcohol abuse (years) & - & $13.1 \pm 4.8$ & $14.1 \pm 4.9$ & $15.7 \pm 5.4$ \\
\hline Ascites (\%) & 0 & $32 \%$ & $61 \%$ & $85 \%$ \\
\hline Esophageal varices (\%) & 0 & $14 \%$ & $45 \%$ & $88 \%$ \\
\hline Encephalopathy (\%) & 0 & $28 \%$ & $151 \%$ & $91 \%$ \\
\hline
\end{tabular}

Table 2. Biochemical characteristics of study and control groups

\begin{tabular}{lcccc}
\hline & \multirow{2}{*}{$\begin{array}{c}\text { Control group } \\
(\mathrm{n}=20)\end{array}$} & $\begin{array}{c}\mathrm{P} \text {-Ch A } \\
(\mathrm{n}=29)\end{array}$ & $\begin{array}{c}\mathrm{P}-\mathrm{Ch} \mathrm{B} \\
(\mathrm{n}=36)\end{array}$ & $\begin{array}{c}\mathrm{P}-\mathrm{Ch} \mathrm{C} \\
(\mathrm{n}=34)\end{array}$ \\
\cline { 3 - 5 } & $0.68 \pm 0.28$ & $2.21 \pm 1.4$ & $4.12 \pm 3.25$ & $7.89 \pm 7.94$ \\
\hline Bilirubin $(\mathrm{mg} / \mathrm{dl})$ & - & $3.27 \pm 0.76$ & $2.78 \pm 0.6$ & $2.44 \pm 0.46$ \\
\hline Albumin $(\mathrm{g} / \mathrm{dl})$ & - & $1.25 \pm 0.27$ & $1.44 \pm 0.29$ & $1.68 \pm 0.41$ \\
\hline INR & - & $32.04 \pm 20.1$ & $23.49 \pm 15.62$ & $39.58 \pm 16.1$ \\
\hline Platelets (G/l) & $226.8 \pm 35.8$ & $186.03 \pm 76.9$ & $123.6 \pm 66.25$ & $114.11 \pm 61.6$ \\
\hline Mean cell volume (fl) & $94.65 \pm 4.45$ & $92.38 \pm 6.25$ & $91.9 \pm 10.08$ & $97.53 \pm 8.02$ \\
\hline Urea (mg/dl) & - & $133.67 \pm 5.3$ & $135.38 \pm 3.6$ & $133.51 \pm 6.63$ \\
\hline Sodium (mmol/l) & $139.82 \pm 3.24$ & $3.88 \pm 0.6$ & $3.94 \pm 0.6$ & $3.3 \pm 0.66$ \\
\hline Potassium (mmol/l) & $4.41 \pm 0.37$ & $14.97 \pm 12.62$ & $19.21 \pm 17.35$ & $20.8 \pm 19.92$ \\
\hline C-reactive protein & $2.11 \pm 1.96$ & & & \\
(mg/l) & & & &
\end{tabular}

biochemical characteristics of the patients are presented in Tables 1 and 2 .

The study protocol was approved by the Ethics Committee. All subjects gave their written informed consent for participation in the study.

\section{Determination of zinc}

Ion chromatography (IC) with UV/VIS detection was used for the determination of zinc ions in the previously mineralized serum samples. During the mineralization procedure, 99\% nitric acid water solution (Sigma- Aldrich, Germany) (1 mL of $\mathrm{HNO}_{3}: 9 \mathrm{~mL} \mathrm{H}_{2} \mathrm{O}$ ) was added to $0.5 \mathrm{ml}$ of serum sample. Microwave-assisted digestion was performed in a NovaWave Microwave Tunnel Digestion System (Scp Science, Montreal, QC, Canada). The mineralized samples were injected onto an analytical column (IonPac CS5A, Dionex, USA). Zinc was detected by measuring the absorbance at $530 \mathrm{~nm}$ of the complex formed with the 4-(2-pyridylazo)resorcinol (PAR) postcolumn reagent. The certified reference material used in this study included Seronorm ${ }^{\mathrm{Tm}}$ Trace Elements Serum L-2 (Billingstad, Norway) - human serum. All reagents were of analytical grade. Deionized water with at least $18.2 \mathrm{M} \Omega-\mathrm{cm}$ resistivity was applied for dilution and preparation of both standard solutions, and eluents. The analyses were carried out using pre-washed polypropylene flasks and vials. The detailed procedure for the preparation of standard solutions, operating IC conditions, and validation of the method applied have been described in previous papers [15]. 


\section{Determination of selenium}

The measurements of selenium in all serum samples were performed with the ContrAA700 high-resolution continuum source graphite tube atomic absorption spectrometer (AAS) (Analytik Jena AG, Jena, Germany). A transversely heated graphite furnace was used as the atomizer. Detailed method parameters were published previously [16].

\section{Determination of FGF-19}

The serum FGF-19 concentration was determined using the Human FGF-19 ELISA Kit (BioVendor, Brno, Czech Republic) according to the manufacturer's procedure. Samples, standards, and quality controls were applied onto pre-coated with polyclonal anti-human FGF-19 antibodies microwells and incubated. Subsequently, biotin-labelled polyclonal anti-human FGF-19 antibodies were added and incubated with captured FGF-19. Unbound antibodies were removed and streptavidin-HRP was added and bound to the biotin-labelled polyclonal anti-human FGF-19 antibodies. During the next step, unbound streptavidin-HRP was removed and the remaining conjugate reacted with HRP added to the wells. A coloured product was formed in proportion to the amount of human FGF-19. The reaction was stopped by addiction of acidic solution, and the absorbance of a yellow product determined using the Epoch Microplate Spectrophotometer (BioTek Instrumentals, Inc., Winooski, VT, USA). Concentrations of FGF-19 in samples were determined using the standard curve.

\section{Determination of endoglin}

ENG concentration was determined using the Enzymelinked Immunosorbent Assay Kit for Endoglin (ENG) (Cloud-Clone Corp., Katy, TX, USA). Standards and samples were applied onto a microtiter plate, which was pre-coated with an antibody specific to ENG. Next, Avidin conjugated to Horseradish Peroxidase (HRP) was added to each microplate well and incubated. During the next step, the TMB substrate solution was added, but only to those wells containing ENG. As a result, biotin-conjugated antibody and enzyme-conjugated Avidin exhibited a colour change. The enzyme-substrate reaction was terminated by the addition of sulphuric acid solution. The colour change was measured spectrophotometrically (wavelength: $450 \mathrm{~nm}$ ) and the concentrations of ENG in the samples determined using a standard curve constructed for standards.

\section{Statistical analysis}

STATISTICA 12 PL was used for data analysis. Continuous variables were expressed as mean \pm standard deviation (SD). Before calculations, variables were checked for normality using the Shapiro-Wilk test; the Brown-Forsythe's test was applied to test equality of variances. To compare the results between more than two groups, the one-way ANOVA was used. The Tukey test was applied for detailed identification of statistically different groups. Correlations among variables were performed using the Pearson's correlation test. Multiple linear regression analysis was performed to determine the potential independent influence of various factors on ENG and FGF-19 concentration. Qualitative variables are shown as indicators of structure (percentage); for intergroup comparisons, the $\chi^{2}$ test was used. For all tests, $\mathrm{p}<0.05$ was considered as statistically significant.

\section{RESULTS}

The highest concentration of selenium was found in the control group, i.e. $0.1 \pm 0.013 \mathrm{mg} / \mathrm{l}$. Lower selenium concentrations were observed in patients with alcoholic liver cirrhosis $-0.068 \pm 0.014 \mathrm{mg} / \mathrm{l}$ in stage $\mathrm{A}, 0.085 \pm 0.016 \mathrm{mg} / \mathrm{l}$ in stage $B$ and $0.072 \pm 0.017 \mathrm{mg} / \mathrm{l}$ in stage $C$ patients (according to the Child-Pugh classification) (Table 3). The post-hoc tests revealed significant differences in selenium concentrations comparing the control group and individual stages of liver cirrhosis (Child-Pugh A, B. C stages; p-value - 0.002; 0.04; 0.001 , respectively). The selenium concentrations in stages $\mathrm{A}, \mathrm{B}$ and $\mathrm{C}$ patients were not significantly different.

Table 3. Concentrations of selenium, zinc, FGF-9 and ENG in cirrhotic patients and controls

\begin{tabular}{|c|c|c|c|c|c|}
\hline & \multirow{2}{*}{$\begin{array}{l}\text { Control } \\
\text { group } \\
(n=20)\end{array}$} & \multicolumn{3}{|c|}{ Alcoholic liver cirrhosis ( $n=99$ ) } & \multirow[b]{2}{*}{$\mathrm{p}^{*}$} \\
\hline & & $\begin{array}{l}\text { P-Ch A } \\
(n=29)\end{array}$ & $\begin{array}{l}\text { P-Ch B } \\
(n=36)\end{array}$ & $\begin{array}{l}\text { P-Ch C } \\
(n=34)\end{array}$ & \\
\hline $\begin{array}{l}\text { Selenium } \\
(\mathrm{mg} / \mathrm{l})\end{array}$ & $0.1 \pm 0.013$ & $0.068 \pm 0.014$ & $0.085 \pm 0.016$ & $0.072 \pm 0.017$ & $<0.001$ \\
\hline Zinc (mg/l) & $1.36 \pm 0.11$ & $1.04 \pm 0.34$ & $0.96 \pm 0.24$ & $1.01 \pm 0.32$ & $<0.001$ \\
\hline $\begin{array}{l}\text { FGF-19 } \\
\text { (pg/ml) }\end{array}$ & $362.1 \pm 197.4$ & $232.9 \pm 107.8$ & $656.2 \pm 756.6$ & $806.9 \pm 650.3$ & 0.01 \\
\hline ENG $(\mathrm{ng} / \mathrm{ml})$ & $3.24 \pm 1.48$ & $6.6 \pm 3.52$ & $7.32 \pm 5.39$ & $7.92 \pm 4.18$ & 0.02 \\
\hline
\end{tabular}

*One-way ANOVA. Results of post-hoc tests discussed in the text.

The concentrations of zinc were significantly lower in Child-Pugh A $(1.04 \pm 0.34 \mathrm{mg} / \mathrm{L}), \mathrm{B}(0.96 \pm 0.24 \mathrm{mg} / \mathrm{L})$, and C $(1.01 \pm 0.32)$ patients, compared to controls $(1.36 \pm 0.11 \mathrm{mg} / \mathrm{L})$. Statistically significant differences were noted between the control group versus Child-Pugh stage A, B and C patients $(\mathrm{p}<0.001 ; \mathrm{p}<0.001 ; \mathrm{p}=0.003$, respectively).

The highest concentration of FGF-19 was found in stage C liver cirrhosis patients $(806.9 \pm 650.3 \mathrm{pg} / \mathrm{ml})$. The significant differences in FGF-19 concentrations were observed between Child-Pugh stage $\mathrm{C}$ patients versus controls $(\mathrm{p}=0.005)$ and stage A patients (compensated cirrhosis) $(\mathrm{p}=0.02)$. Moreover, significant differences were found between the Child-Pugh stage B subgroup and controls $(\mathrm{p}=0.02)$.

The highest concentration of ENG was demonstrated in the control group $(3.24 \pm 148 \mathrm{ng} / \mathrm{ml})$ while the lowest in patients with decompensated cirrhosis $(7.32 \pm 5.39 \mathrm{ng} / \mathrm{ml}$ and $7.92 \pm 4.18 \mathrm{ng} / \mathrm{ml}$ for Child-Pugh stage $B$ and $C$, respectively. The test of multiple comparisons revealed that the concentration of ENG was significantly different between the control group and patients with stage $\mathrm{B}$ cirrhosis $(\mathrm{p}=0.03)$ as well as patients with stage $C$ cirrhosis $(p=0.02)$. The differences between the control group and patients with stage A cirrhosis were not significant; likewise, the differences among stages of liver cirrhosis were not significant.

There were no significant correlations between the concentration of FGF-19 versus the concentration of selenium $(r=0.16 ; p=0.25)$ and of zinc $(r=0.12 ; p=0.39)$. However, a reverse correlation was found between the concentration of ENG and the concentration of selenium $(r=-0.26 ; p=0.4)$, but not the concentration of zinc ( $\mathrm{p}=-0.17 ; \mathrm{p}=0.2)$.

Use of the multiple-variable model designed using multiple linear regression demonstrated that the independent factors affecting the concentration of ENG were the concentration of bilirubin ( $\mathrm{p}=0.02)$, INR $(\mathrm{p}=0.01)$ and duration of alcohol abuse $(\mathrm{p}=0.02)$ (Table 4$)$. The independent determinants of 
Table 4. Factors influencing FGF-19 and ENG concentrations determined by multiple linear regression

\begin{tabular}{|c|c|c|c|c|c|c|}
\hline $\begin{array}{l}\text { Dependent } \\
\text { variables }\end{array}$ & $\begin{array}{l}\text { Independents } \\
\text { variables }\end{array}$ & $b^{*}$ & SE & $\mathrm{b}$ & SE & $\mathrm{p}$ \\
\hline & Intercept & & & 15.15 & 3.28 & $<0.0001$ \\
\hline & drinking period & 0.21 & 0.09 & 0.15 & 0.07 & 0.02 \\
\hline \multirow[t]{4}{*}{ ENG } & Bilirubin & 0.27 & 0.11 & 0.17 & 0.07 & 0.02 \\
\hline & INR & 0.26 & 0.11 & 2.83 & 1.12 & 0.01 \\
\hline & \multicolumn{6}{|c|}{ Model: $\mathrm{R}=0.45$, adjusted $\mathrm{R}^{2}=0.2, \mathrm{p}=0.01$} \\
\hline & Pugh-Child class & 0.23 & 0.11 & 95.38 & 46.11 & 0.04 \\
\hline \multirow[t]{2}{*}{ FGF-19 } & INR & 0.23 & 0.10 & 325.2 & 150.3 & 0.03 \\
\hline & \multicolumn{6}{|c|}{ Model: $\mathrm{R}=0.37$, adjusted $\mathrm{R}^{2}=0.14, \mathrm{p}=0.01$} \\
\hline
\end{tabular}

Abbreviations: $\mathrm{b}^{*}$ - standardized coefficient of regression; $\mathrm{b}$ - coefficient of regression; SE standard error; $\mathrm{R}$ - coefficient of multiple correlation; $\mathrm{R}^{2}$ - coefficient of determination.

FGF-19 concentrations were found to be the stage (severity) of liver cirrhosis (Pugh-Child) $(\mathrm{p}=0.04)$ and INR $(\mathrm{p}=0.03)$. The values of corrected coefficients of determination for both the above models were relatively low ( 0.2 and 0.14 , respectively); it should be stressed, however, that these were the optimal obtainable models.

\section{DISCUSSION}

Our study findings demonstrated reduced serum concentrations of microelements, i.e. selenium and zinc, in patients with alcoholic liver cirrhosis, which is consistent with numerous earlier publications. The concentrations of selenium and zinc were reduced in all patients with liver cirrhosis, irrespective of the stage of disease.

The deficiency of selenium is common in patients with liver cirrhosis [17]. Many experimental studies have revealed the relationship between the deficiency of selenium and pathogenetic processes involved in the development of liver cirrhosis. Long-term deficiency of selenium can lead to liver damage in the mechanism associated with impaired fibrogenesis and fibrolysis [18]. Selenium supplementation inhibits the expression of pro-inflammatory factors TNF- $\alpha$ and IL- $1 \beta$ and promotes the production of anti-inflammatory cytokine IL-10 in the liver with acute alcoholism-suppressed caspases, proaptototic enzyme [19].

Moreover, there are literature reports providing grounds to consider selenium compounds as hepatoprotective factors. Ozkol et al. in their experimental model have shown that supplementation of selenium (alone or in combination with vitamin $\mathrm{E}$ and $\mathrm{N}$-acetylcysteine) exerts hepatoprotective effects in rats exposed to ethanol [20]. The authors have demonstrated that the positive effects of selenium are likely to result from its antioxidative and neutralising action against free radicals. According to Kalishwaralal et al., selenium compounds (sodium selenite/selenium nanoparticles) showed cardioprotective effects in Danio rerio embryos exposed to ethanol. Selenium and SeNPs were found to reduce the $1 \%$ ethanol-induced oxidative damage through scavenging intracellular reactive oxygen species [21].

Zinc is a microelement involved in cellular metabolism. More than 300 enzymes have zinc ions [22]. In 1940, Keilin and Mann were the first to detect the presence of zinc in the structure of carbonic anhydrase [23], which is involved in the regulation of acid-base balance in the lungs and kidneys. It is noteworthy that zinc is found in the structure of superoxide dismutase, the enzyme involved in oxy-redox reactions [24]. Zinc is a functional component of alcohol dehydrogenase [25]. It stabilises the structure of zinc fingers, plays an important role in DNA transcription and repair, division and apoptosis of cells. Zinc fingers stabilise the structure of transcription factors, steroid hormones, the thyroid and vitamin D [26]. The deficiency of zinc induces oxidative stress, causing hepatitis and decreasing the response to infections and toxic substances [27].

Walker et al. have shown that the plasma zinc level is significantly lower in patients with liver cirrhosis than in controls, and urinary zinc excretion is increased in cirrhosis [28]. Our study findings are comparable, showing that the concentration of zinc was statistically lower in the group of patients with stage A, B and C alcoholic liver cirrhosis, compared to controls.

The reduced concentrations of zinc in liver diseases is caused by deficiency of zinc in diets, reduced concentration of albumins and impaired absorption of zinc induced by alcohol. The use of diuretics in the treatment of ascites in the course of liver cirrhosis increased renal zinc excretion and reduced the capacity of albumin to bind zinc. In their interesting publication, Omran et al. demonstrated reduced concentrations of zinc in $\mathrm{HCV}$-positive patients with liver cirrhosis, which deepened with the progression of fibrosis. Serum zinc levels have been shown to have a negative significant correlation with INR and with FibroScan scores, but no correlation to bilirubin, ALT, AST, or albumin.

To-date, there are no reports regarding ENG in alcoholic liver cirrhosis. To the best of our knowledge, the present study is the first to describe significantly elevated concentrations of ENG in patients with advanced liver cirrhosis (ChildPugh stage B-C), compared to patients without cirrhosis. Yagmur et al. have revealed that the concentration of ENG is statistically increased in a group of patients with liver cirrhosis, compared to controls. However, their study included patients with cirrhosis of various etiologies, and not only with alcoholic cirrhosis, as was the case in our study. Moreover, the authors have shown that the concentration of ENG is significantly higher in patients with hepatocellular carcinoma developing during liver cirrhosis, compared to patients with liver cirrhosis. Furthermore, a positive correlation has been observed between the concentration of ENG and $\alpha$-fetoprotein [8]. Kwan et al. have described a potential role of ENG in initiation of fibrosis in patients with liver cirrhosis in the course of HCV infection [29]. ENG is an accessory receptor for transforming growth factor- $\beta$ involved in the pathogenesis of fibrosis. Activated hepatic stellate cells express ENG. Clemente et al. have showed that serum levels of ENG are significantly higher in patients with advanced fibrosis than in those with early fibrosis in chronic hepatitis $C$ patients [30].

As mentioned above, the majority of researchers focus on antioxidative and antiapoptotic potential of selenium in liver damage and cirrhosis. The available literature lacks reports regarding the relation of selenium and ENG, which is a proangiogenic and profibrotic cytokine. The majority of papers have focused on the role of selenium in inhibiting angiogenesis in malignant tumours [31]. Only a few studies have been devoted to interrelations between zinc and ENG. Rezende et al. demonstrated a negative correlation between the concentration of zinc and ENG in the serum of pregnant patients $(\mathrm{r}=0.43 ; \mathrm{p}=0.003)$ [32]. 
The studies about the role of FGF-19 in liver diseases have mainly been performed in patients with primary biliary cirrhosis. FGF-19 is considered a polypeptide hormone involved in the transmission of signals in the intestinalhepatic axis regulating the synthesis of bile acids. Wunsch et al. have demonstrated that the hepatic expression of FGF-19 is increased in patients with primary biliary cirrhosis and is correlated with the stage of disease [9]. In our study, statistically significantly elevated concentrations of this cytokine were reported for the first time in patients with alcoholic liver cirrhosis. The highest concentrations were noted in patients with decompensated liver cirrhosis, which is likely to imply the involvement of FGF-19 in the pathogenesis of liver fibrosis and development of cirrhosis in alcoholic liver disease.

Our findings did not demonstrate significant correlations between the concentration of FGF-19 and the concentration of selenium; on the other hand, a negative correlation was observed between the concentration of selenium and ENG $(\mathrm{r}=-0.26 ; \mathrm{p}=0.4)$. However, the independent relationship of selenium and concentration of ENG was not confirmed in the multiple-variable model. Moreover, the concentration of zinc did not significantly correlate with the levels of ENG and FGF-19. Further studies are needed regarding the effects of zinc and selenium on other cytokines and/or cells involved in the progression of cirrhosis.

\section{CONCLUSIONS}

Reduced concentrations of zinc and selenium in serum of patients with alcoholic liver cirrhosis correlate with the severity of the disease, but are not independently related to concentrations of FGF-19 and ENG. Further studies are required to widen our knowledge regarding the interactions of zinc and selenium in cells and cytokines involved in the pathogenesis of alcoholic liver cirrhosis.

\section{Acknowledgments}

The authors express their thanks Anna Misiuna M.A. for providing medical writing services on behalf of the Medical University of Lublin, Poland.

\section{REFERENCES}

1. Blachier M, Leleu H, Peck-Radosavljevic M, Valla D-C, RoudotThoraval F. The burden of liver disease in Europe: A review of available epidemiological data. J Hepatol. 2013; 25(3): 593-608.

2. World Health Organization. European status report on alcohol and health: World Health Organization. Regional Office for Europe, 2010.

3. Mokdad A, Lopez A, Shahraz S, et al. Liver cirrhosis mortality in 187 countries between 1980 and 2010: a systematic analysis. BMC Medicine 2014; 12: 145.

4. Zhou W-C, Zhang Q-B, Qiao L. Pathogenesis of liver cirrhosis. World J Gastroenterol. 2014; 20(23): 7312-7324.

5. Robak E, Gerlicz Z. Wybrane cytokiny proangiogenne w twardzinie układowej._Postepy Hig Med Dosw. 2014; 68: 1472-1482

6. Westphal J, Willems H, Schalwijk C, Ruiter D, de Waal R. A new 180$\mathrm{kDa}$ dermal endothelial cell activation antigen: in vitro and in situ characteristics. J Invest Dermatol. 1993; 100: 27-34.

7. Meurer S, Alsamman M, Sahin H, Wasmuth H, Kisseleva T, Brenner $\mathrm{D}$, et al. Overexpression of endoglin modulates TGF- $\beta 1$-signalling pathways in a novel immortalized mouse hepatic stellate cell line. PLoS One. 2013; 8(2): e56116.

8. Yagmur E, Rizk M, Stanzel S, Hellerbrand C, Lammert F, Trautwein C, et al. Elevation of endoglin (CD105) concentrations in serum of patients with liver cirrhosis and carcinoma. Eur J Gastroenterol Hepatol. 2007; 19(9): 755-761.
9. Wunsch E, et al. Expression of hepatic Fibroblast Growth Factor 19 is enhanced in Primary Biliary Cirrhosis and correlates with severity of the disease. Sci Rep. 2015; 5: 13462.

10. Hyeon J, Ahn S, Lee J, Song D, Park C. Expression of fibroblast growth factor 19 is associated with recurrence and poor prognosis of hepatocellular carcinoma. Dig Dis Sci. 2013; 58(7): 1916-1922.

11. Prystupa A, Błażewicz A, Kiciński P, Sak JJ, Niedziałek J, Załuska W. Serum Concentrations of Selected Heavy Metals in Patients with Alcoholic Liver Cirrhosis from the Lublin Region in Eastern Poland. Int J Environ Res Public Health. 2016; 13(6): E582.

12. Omran D, Darweesh S, Fouad H, Mahmoud M, Saif S, Fared A, et al. Serum Zinc Deficiency and its Relation to Liver Fibrosis in Chronic HCV: a Real-Life Egyptian Study. Biol Trace Elem Res. 2017; doi: 10.1007/s12011-017-0938-x.

13. Burk R, Hill K, Motley A, Byrne D, Norsworthy B. Selenium deficiency occurs in some patients with moderate-to-severe cirrhosis and can be corrected by administration of selenate but not selenomethionine: a randomized controlled trial. Am J Clin Nutr. 2015; 102(5): 1126-1133.

14. Pugh R, Murray-Lyon I, Dawson J, Pietroni M, et al. Transection of the oesophagus for bleeding oesophageal varices. Br J Surg. 1973, 60(8): 646-9.

15. Błażewicz A, Baj T, Świeboda R, Świątek Ł. Determination of heavy metals in aerial part of hyssop (Hyssopus officinalis L.) using high performance ion chromatography with the aid of a linear and nonlinear weighted least-squares regression model. Pol J Environ Stud. 2007; 16: 191-198.

16. Błażewicz A, Klatka M, Astel A, Korona-Glowniak I, Dolliver W, Szwerc W, et al. Serum and urinary selenium levels in obese children: a crosssectional study. J Trace Elem Med Biol. 2015; 29: 116-122.

17. Burk R, Hill K, Motley A, Byrne D, Norsworthy B. Selenium deficiency occurs in some patients with moderate-to-severe cirrhosis and can be corrected by administration of selenate but not selenomethionine: a randomized controlled trial. Am J Clin Nutr. 2015; 102(5): 1126-1133.

18. Han J, Liang H, Yi J, Tan W, He S, Wang S, et al. Long-Term SeleniumDeficient Diet Induces Liver Damage by Altering Hepatocyte Ultrastructure and MMP1/3 and TIMP1/3 Expression in Growing Rats. Biol Trace Elem Res. 2017; 175(2): 396-404.

19. Zhang Z, Guo Y, Qiu C, Deng G, Guo M. Protective Action of SeSupplement Against Acute Alcoholism Is Regulated by Selenoprotein $\mathrm{P}$ (SelP) in the Liver. Biol Trace Elem Res. 2017; 175(2): 375-387.

20. Ozkol H, Bulut G, Balahoroglu R, Tuluce Y, Ozkol HU. Protective Effects of Selenium, N-Acetylcysteine and Vitamin E Against Acute Ethanol Intoxication in Rats. Biol Trace Elem Res. 2017; 175(1): 177-185.

21. Kalishwaralal K, Jeyabharathi S, Sundar K, Muthukumaran A. Sodium selenite/selenium nanoparticles (SeNPs) protect cardiomyoblasts and zebrafish embryos against ethanol induced oxidative stress. J Trace Elem Med Biol. 2015; 32: 135-144.

22. Vallee B, Falchuk K. The biochemical basis of zinc physiology. Physiol Rev. 1993; 73: 79-118.

23. Keilin D, Mann T. Carbonic anhydrase. Nature. 1939; 144: 442-443.

24. Oteiza P. Zinc and the modulation of redox homeostasis. Free Radical Biology and Medicine. 2012; 53(9): 1748-1759.

25. Vallee B, Hoch F. Zinc, a component of yeast alcohol dehydrogenase. Proc Natl Acad Sci USA. 1955; 15, 41(6): 327-338.

26. Krishna S, Majumdar I, Grishin N. Structural classification of zin fingers: survey and summary. Nucleic Acids Res. 2003, 31(2): 532-550.

27. Parsons S, Di Silvestro R. Effects of mild zinc deficiency, plus or minus an acute-phase response on galactosamine-induced hepatitis in rats. Br Nutr. 1994; 72: 611-618.

28. Walker B, Dawson J, Kelleher J, Losowsky M. Plasma and urinary zinc in patients with malabsorption syndromes or hepatic cirrhosis. Gut. 1973; 14(12): 943-948.

29. Kwon Y, Sasaki R, Meyer K, Ray R. Hepatitis C virus core protein modulates endolglin (CD105) signalling pathway for liver pathogenesis. J Virol. 2017; 9. pii: JVI.01235-17. doi: 10.1128/JVI.01235-17.

30. Clemente M, Núñez O, Lorente R, Rincón D, Matilla A, Salcedo M, et al. Increased intrahepatic and circulating levels of endoglin, a TGFbetal co-receptor, in patients with chronic hepatitis $C$ virus infection: relationship to histological and serum markers of hepatic fibrosis. J Viral Hepat. 2006; 13(9): 625-632.

31. Saghiri M, Asatourian A, Orangi J, Sorenson C, Sheibani N. Functional role of inorganic trace elements in angiogenesis - Part I: N, Fe, Se, P, Au, and Ca. Crit Rev Oncol Hematol. 2015; 96(1): 129-142.

32. Rezende V, Barbosa F Jr, Palei A, Cavalli R, Tanus-Santos J, Sandrim V. Correlations among antiangiogenic factors and trace elements in hypertensive disorders of pregnancy. J Trace Elem Med Biol. 2015; 29: $130-135$. 